\title{
EFICIENCIA MUNICIPAL Y SERVICIOS PÚBLICOS EN MICHOACÁN, MÉXICO*
}

\author{
Julio César Morán Figueroa ${ }^{a}$ \\ Francisco Javier Ayvar Campos
}

* DOI: https://doi.org/10.18601/01245996.v20n43.12 Recepción: 01-062019, modificación final: 10-04-2020, aceptación: 06-05-2020. Sugerencia de citación: Morán Figueroa, J. C. y Ayvar Campos, F. J. (2020). Eficiencia municipal y servicios públicos en Michoacán, México. Revista de Economía Institucional, 22(43), 277-299.

a Maestro en Políticas Públicas. Estudiante de doctorado en Políticas Púbicas, Instituto de Investigaciones Económicas y Empresariales, Universidad Michoacana de San Nicolás de Hidalgo, México, [moran_figueroa@hotmail. com], [https://orcid.org/0000-0002-9344-3067].

b Doctor en Ciencias del Desarrollo Regional. Profesor investigador, Instituto de Investigaciones Económicas y Empresariales, Universidad Michoacana de San Nicolás de Hidalgo, México, [fayvar@umich.mx], [https://orcid. org/0000-0001-7342-4451]. 


\section{Eficiencia municipal y servicios públicos en Michoacán, México}

Resumen. Este trabajo evalúa el uso de los recursos para proveer servicios públicos en el estado de Michoacán, en 2014, usando el análisis envolvente de datos y considerando los insumos (recursos) y productos (servicios públicos) del municipio. Los resultados indican que solo 22 municipios proporcionaron servicios públicos de manera eficiente, y aunque los recursos financieros de los gobiernos locales han aumentado se emplean de manera ineficiente.

Palabras clave: eficiencia, servicios públicos, DEA, municipio; JEL: H21, H41

\section{Municipal efficiency and public services in Michoacán, Mexico}

Abstract. This document addresses the use of resources for local public services in the state of Michoacán, Mexico, in 2014. The state of production is analyzed through its inputs (resources) and outputs (public services). The results show that only twentytwo municipalities were efficient in the provision of public services. Although the financial resources for the local governements have increased, these resources are employed inefficiently.

Keywords: efficiency, public services, DEA, municipality; JEL: H21, H41

\section{Eficiência municipal e serviços públicos em Michoacán, México}

Resumo. Este trabalho avalia o uso de recursos para a prestação de serviços públicos no estado de Michoacán, em 2014, usando a análise envolvente de dados e considerando as entradas (recursos) e produtos (serviços públicos) do município. Os resultados indicam que apenas 22 municípios prestaram serviços públicos com eficiência e, embora os recursos financeiros dos governos locais tenham aumentado, eles são usados de maneira ineficiente.

Palavras-chave: eficiência, serviços públicos, DEA, municipio; JEL: H21, H41 
H. nel periodo 1980-2000 hubo una reestructuración institucional Por un lado, varias reformas constitucionales dieron base y certeza jurídica a los gobiernos locales como proveedores de servicios públi$\cos ^{1}$. Por el otro, la reforma del pacto fiscal permitió que los recursos públicos llegaran en forma continua a los estados y municipios ${ }^{2}$.

Para aumentar las facultades de los municipios se argumenta que el municipio es el nivel de gobierno más cercano a la ciudadanía y conoce mejor las necesidades de sus habitantes, de modo que puede ser más responsable, estar más interesado en atenderlos y proporcionarles servicios al menos con la misma eficiencia que otros niveles de gobierno (Broid, 2010). No obstante, la evidencia empírica muestra que pese a esta atribución de funciones y al gran aumento sustancioso de los recursos, aún persisten problemas de calidad y cobertura de los servicios públicos básicos (Cabrero, 2011).

Esta situación hace necesario medir la eficiencia de los gobiernos municipales en los gastos para proporcionar servicios básicos (Olivera y Cano, 2012). Desde un punto de vista económico se puede concebir el municipio como una unidad de producción (DMU) que utiliza recursos para suministrar productos que satisfacen las necesidades de los habitantes (Balaguer, 2004; Herrera y Málaga, 2007; Herrera y Fracke, 2007). Este trabajo evalúa la eficiencia de los gobiernos municipales del estado de Michoacán en los gastos de inversión y servicios personales para prestar los servicios de alumbrado público, recolección de basuras y seguridad pública en 2014. Un servicio público es

toda actividad técnica destinada a satisfacer una necesidad de carácter general, cuyo cumplimiento uniforme y continuo deba ser permanente-

${ }^{1}$ Se hicieron tres reformas al artículo 115 de la Constitución Política. La primera, en 1983, añadió el apartado III, por el cual los servicios públicos son responsabilidad de los gobiernos municipales. La segunda, en 1987, suprimió las disposiciones sobre los estados y solo dejó las disposiciones municipales. La tercera, en 1999, reconoció al municipio como ente de gobierno y no como extensión de las estructuras estatales (IDM, 1999; García, 2011; H. Congreso de la Unión, 1978 y 2019).

${ }^{2}$ En 1980 entró en vigor el Sistema Nacional de Coordinación Fiscal, que modernizó la distribución del ingreso entre los tres órdenes de gobierno. En lo que respecta a los ingresos municipales, en 1981-1982 se incorporó el Fondo de Fomento Municipal, que aumentó en los años ochenta hasta llegar al 1\% de la Recaudación Federal Participable en 1996. Las participaciones federales a los municipios se incorporaron en 1997, con el Fondo para la Infraestructura Social Municipal, y en 1999, con el Fondo para el Fortalecimiento de los Municipios y las Demarcaciones del Distrito Federal (Herrera, Maya y Huerta, 2017; Astudillo, 2005). 
mente asegurado, regulado y controlado por los gobernantes, con sujeción a un régimen jurídico, ya por medio de la Administración Pública o bien mediante particulares facultados para ello por la autoridad competente, en beneficio indiscriminado de toda persona (Fernández, 2002, p. 121).

El éxito o el fracaso en la provisión de servicios públicos se determina evaluando o examinando de modo sistemático las acciones, programas y políticas y sus efectos de corto, mediano y largo plazos (Starling, 1988). Si se busca saber hasta qué punto la política o el programa logran sus objetivos se trata de una evaluación de eficacia; y si quiere conocer el costo de conseguirlos (en tiempo, recursos humanos, materiales y monetarios) se trata de una evaluación de eficiencia (Osuna, 2013).

Para medir la eficiencia utilizamos el análisis envolvente de datos (DEA), que compara los resultados de distintas unidades de producción (Farrell, 1957; Charnes, Cooper y Rhodes, 1978; Bemowski, 1991) y se suele utilizar para medir la eficiencia de los gobiernos locales en la prestación de servicios públicos (Giménez y Pior, 2003; Balaguer, 2004; Herrera y Fracke, 2007).

El artículo consta de cinco secciones. En la primera se hace un diagnóstico de la situación actual de los servicios públicos en $\mathrm{Mi}$ choacán; en la segunda se analizan los aspectos teóricos de los servicios públicos y del DEA; en la tercera se describe el método para construir el modelo DEA que se usa para estimar la eficiencia en la prestación de servicios públicos. En la cuarta apartado se comentan los resultados del modelo y se identifica los municipios eficientes. Por último se exponen las conclusiones generales.

\section{LOS SERVICIOS PÚBLICOS MUNICIPALES EN MICHOACÁN}

Por mandato constitucional, los gobiernos municipales de Michoacán son responsables de proporcionar diversos servicios públicos a los habitantes de sus jurisdicciones ${ }^{3}$ (Fernández, 2002). En el estado viven

${ }^{3}$ La Fracción III del Artículo 115 de la Constitución Política de los Estados Unidos Mexicanos y la Fracción V del Artículo 123 de la Constitución Política del Estado de Michoacán establecen los servicios públicos que están a cargo de los municipios. E1 artículo 71 de la Ley Orgánica Municipal del Estado de Michoacán enumera los siguientes: I. Agua potable, drenaje, alcantarillado, tratamiento y disposición de sus aguas residuales; II. Alumbrado público; III. Limpia, recolección, traslado, tratamiento y disposición final de residuos; IV. Mercados y centrales de abastos; V. Panteones; VI. Rastro; VII. Calles, parques, y jardines; y VIII. Policía preventiva municipal y tránsito (H. Congreso de la Unión, 1978 y 2019; H. Congreso de Michoacán, 2001 y 2019). 
4.584.471 de personas, en 113 municipios; 11 de ellos concentran el 50,3\% de la población, entre los que se destacan Morelia con 784.785 y Uruapan con 334.749 personas. Seguidos por Zamora y Lázaro Cárdenas con 196.208 y 183.185, respectivamente (Inegi, 2015).

El hecho de que 11 municipios concentren la mitad de la población no implica que no esté dispersa en el territorio. Según el Censo de Población y Vivienda de 2010, en Michoacán existen 9.612 centros de población (ver cuadro $1 \mathrm{del}$ anexo) y muchos de ellos son pequeñas rancherías con apenas un centenar de habitantes. Como ejemplos de esta dispersión cabe mencionar que en Coalcomán hay 1.222 centros poblacionales; en Aquila, 985; en Arteaga, 909; en Turicato, 767; en Tzitzio, 707, y en Tiquicheo, 669 (Inegi, 2010).

Esta población dispersa requiere y demanda servicios públicos municipales. Un diagnóstico integral de la provisión de estos servicios públicos debe considerar dos aspectos fundamentales: los recursos que se emplean y los servicios que se prestan a la ciudadanía.

\section{LOS RECURSOS FINANCIEROS DE LOS MUNICIPIOS DE MICHOACÁN}

La provisión de servicios públicos municipales requiere el uso de recursos humanos, administrativos y materiales, y la existencia de un área administrativa que coordine las acciones de gobierno (Fernández, 2002). La base de esta estructura administrativa es una hacienda municipal independiente que tiene autonomía para programar los recursos financieros necesarios para llevar a cabo las acciones y políticas del gobierno municipal (Raich, 2000).

En 2014, los municipios de Michoacán ejecutaron en conjunto un presupuesto de algo más de 13.412 millones de pesos, equivalentes a 2.930 pesos per cápita (Inegi, 2015 y 2017). Los mayores presupuestos fueron Morelia, 2.211 millones de pesos; Uruapan, 752 millones y Lázaro Cárdenas, 658 millones. Los municipios que recibieron menores presupuestos fueron Chucándiro, 29 millones; Huiramba, 27 millones, y Zináparo, 21 millones (Inafed, 2015).

En los presupuestos municipales existen dos grandes rubros: el gasto de inversión y el gasto corriente. $\mathrm{El}$ primero incluye todos los gastos destinados a ampliar o mejorar los bienes y propiedades del municipio; y el segundo, todos los gastos que no tienen como contrapartida la creación de un activo (ASM, 2011). En 2014 el gasto corriente de los 113 municipios fue del 73,9\% del total de los recursos y el 26,1\% restante se dedicó a inversión (Inegi, 2017).

Entre 1990 y 2014, en el contexto del Nuevo Sistema de Coordinación Fiscal, los recursos municipales del estado tuvieron un aumento 
neto promedio de un $720 \%$. Aunque este aumento fue muy dispar, pues mientras que municipios como La Piedad o Marcos Castellanos apenas duplicaron sus recursos, en Lázaro Cárdenas aumentaron el 3.342\%, debido al boom demográfico de los últimos 30 años (ver cuadro 1 del anexo).

\section{SituACión ACTUAL de LOS SERVICIOS PÚBlicos EN MichoACÁN}

La provisión de servicios públicos ha avanzado notablemente en México y en Michoacán durante los últimos 30 años; aunque falta mucho por hacer (García y Gómez, 2011). En la prestación de los servicios se pueden considerar la cobertura y las unidades físicas que los proporcionan (Balaguer, 2004).

En materia de alumbrado público existen 362.456 puntos de luz distribuidos en el estado, una luminaria por cada 12 habitantes (Inegi, 2015). En recolección de basuras, 73 municipios reportaron que en 2014 atendían al 100\% de la población, y otros 13 a un poco más del 90\%. Los que atendían a menos del 90\% son municipios con un alto número de centros poblacionales, como Tancítaro, Aquila y Tiquicheo, donde el servicio no llega a las comunidades rurales más apartadas. En 2014, solo 13 municipios reportaron que daban mantenimiento al $100 \%$ de las calles y vías, de modo que este es el servicio público con más deficiencias (Inegi, 2015).

En los servicios proporcionados en un espacio físico, en 2014 se reportaron 170 mercados municipales, 144 mataderos municipales y 768 cementerios, la mayoría de carácter municipal, algunos privados y otros comunales. Además, existían 2.985 parques y jardines públicos al cuidado de administraciones municipales, uno por cada 1.536 habitantes (Inegi, 2015).

En materia de seguridad pública el marco jurídico establece que las policías municipales solo deben enfrentar delitos de fuero común, como robos, riñas o alteraciones del orden público. En 2014 había 274 comandancias y módulos de seguridad en el estado; que reportaron 60.017 intervenciones para restaurar el orden público, es decir, 164 al día (Inegi, 2015).

\section{SERVICIOS PÚBLICOS Y EFICIENCIA. ASPECTOS TEÓRICOS}

\section{LA PROVISIÓN DE SERVICIOS PÚBLICOS}

Los servicios públicos son "actividades técnicas que el estado declara de interés general porque satisfacen necesidades colectivas en el entendido de que la satisfacción se realiza a través de pres- 
taciones directas e individualizadas a los usuarios de un servicio" (Valencia, 2006, p, 214). Para Antúnez y Galilea (2003) son un conjunto de instrumentos y medidas de los que debe disponer la ciudadanía para el pleno desarrollo de su potencial humano, social y económico. Para Jaramillo, Monroy y Villamizar (2005), son actividades destinadas a satisfacer necesidades básicas de la comunidad, suministradas de manera metódica, uniforme y persistente, bien sea en forma gratuita o pagando una tarifa (Fernández, 2002; Andrade, 2006). Los servicios públicos deben ser generales o universales, es decir, que toda persona pueda utilizarlos si lo desea. Además, deben prestarse sin discriminación de ningún tipo (García y Gómez, 2011).

Las reformas de la administración pública se basan en la idea de que el gobierno municipal debe para proporcionar ciertos servicios porque conoce mejor las necesidades de los pobladores y que la administración municipal los provee con igual o más eficiencia que el gobierno central (Oates, 1999). Por su parte, Olson (1969) argumenta que la jurisdicción política y el área cubierta por el servicio público deben coincidir para que el beneficio marginal sea igual al costo marginal y así se evite el problema del gorrón.

Los ciudadanos son cada vez más conscientes de sus derechos, entre ellos a buenos servicios públicos; y las administraciones saben que deben dar cumplimiento a esos derechos y a las expectativas ciudadanas, aunque no sean de fácil identificación (Denhardt y Denhardt, 2003; Aberbach y Christensen, 2005), pues no solo se refieren a la provisión de los servicios sino también a la eficiencia en su prestación (Olivera y Cano, 2012). Una manera de estimar esta eficiencia es el análisis comparativo del uso de los recursos entre municipios, para lo cual se utiliza el DEA (Balaguer, 2004; Herrera y Málaga, 2007; Herrera y Fracke, 2007).

\section{LA EFICIENCIA Y EL ANÁLISIS ENVOLVENTE DE DATOS}

En 1957, Michael Farrell publicó un trabajo que trazó las pautas de la investigación sobre la eficiencia. Allí estimó la isocuanta de la empresa más eficiente, y luego determinó la distancia entre ella y la de las empresas las ineficientes (Delfín y Navarro, 2014). Este método fue extendido por Bradford, Malt y Oates (1969), Aigner, Lovell y Schmidt (1977), y Charnes et al. (1978), para estudiar la eficiencia de manera empírica aplicando métodos paramétricos (deterministas y estocásticos y no paramétricos (DEA). Los métodos paramétricos requieren especificar una función de producción y emplear técnicas 
econométricas; los no paramétricos usan algoritmos de programación lineal y benchmarking (Budnick, 2007).

El DEA es una técnica no paramétrica y determinista en la que se construye una frontera de eficiencia a partir de los datos de las entidades que se estudian, las unidades de toma de decisiones (DMU, por sus siglas en inglés), estimando el valor de los insumos y productos que maximizan el grado de eficiencia en la producción (Seiford y Thrall, 1990; Bosch, Navarro y Giovagnoli, 1999; Coll y Blasco, 2006; Restrepo y Villegas, 2008). Para cada DMU se construye una frontera de producción empírica a partir de sus insumos y sus productos, luego se identifican las unidades eficientes y no eficientes, asignándoles una puntuación de eficiencia relativa (Navarro, Ayvar y Giménez, 2016). La eficiencia de cada DMU se mide como su con respecto a la frontera (Navarro, 2005; Gómez, 2014). La DMU que se emplea como referente proporciona información para guiar las decisiones de las DMU ineficientes de modo que mejoren su eficiencia (Seijas, 2005; Herrera y Málaga, 2007).

Existen cuatro tipos de modelos DEA: de rendimientos constantes a escala (RCS), de rendimientos variables a escala (RVS), el aditivo y el multiplicativo (Navarro et al., 2016), que pueden tener tres orientaciones: insumo, producto e insumo-producto o no orientado (Silva, Castro y Thanassoulis, 2003).

\section{SERVICIOS PÚBLICOS Y ANÁLISIS ENVOLVENTE DE DATOS}

E1 DEA se suele utilizar para evaluar la eficiencia de los gobiernos locales en la provisión de servicios públicos. Los trabajos pioneros de Vanden, Tulkens y Jamar (1993), De Borger et al. (1994) y De Borger y Kerstens (1996) analizaron el uso del gasto corriente en la prestación de servicios públicos en varios municipios de Bélgica. Por su parte, Taïrou (2000) evaluó la eficiencia de los municipios franceses utilizando el gasto corriente como input y los servicios municipales como productos.

En España existen numerosos trabajos que emplean el DEA para analizar la eficiencia de costos y las especificaciones de los productos ${ }^{4}$. En Chile, Pacheco, Sánchez y Villena (2013) aplicaron el DEA para medir la eficiencia de los gobiernos municipales. En Perú se destacan los trabajos de Herrera y Málaga (2007) y Herrera y Fracke (2007), que analizaron la eficiencia del gasto municipal y sus determinantes con métodos paramétricos y no paramétricos. Cabe mencionar tam-

${ }^{4}$ Ver, p. ej., Prieto y Zoflo (2001), Balaguer, Prior y Tortosa (2007) y Selva y Sanz (2015). 
bién los estudios de Worthington y Dollery (2000), Moore, Nolan y Sepal (2005), Afonso y Fernandes (2006) y Bellido (2010), que determinaron la incidencia del uso eficiente de los recursos en la provisión de servicios municipales.

\section{METODOLOGÍA}

En este trabajo se utiliza el DEA para determinar la eficiencia de los municipios de Michoacán en la provisión de servicios municipales. Una de las bondades de esta técnica es que no se necesita construir una función predeterminada y, así, evita errores de especificación. Además, se requiere poca información de las variables de entrada y salida, lo que es muy útil cuando se analizan servicios públicos, donde es difícil determinar el precio de producción o de mercado. Por último, el benchmarking permite comparar el desempeño de las DMU ineficientes y las eficientes, el cual poder servir de base para diseñar estrategias de mejoramiento (Bemowski, 1991; Coll y Blasco, 2006).

\section{SELECCIÓN DE PRODUCTOS E INSUMOS}

La selección de las variables del modelo se llevó a cabo en tres etapas: primero se revisó la literatura sobre la eficiencia de los servicios públicos de gobiernos locales ${ }^{5}$, basados en el DEA, para determinar los productos e insumos posibles. Luego se verificó que hubiera datos de estas variables en las bases estadísticas oficiales. Y, por último, se realizó el análisis factorial con los datos disponibles para elegir las variables estadísticamente más representativas.

Con base en la revisión de la literatura y la disponibilidad de datos se obtuvieron 9 productos y 8 insumos 6 . Para seleccionar las variables de entrada y de salida más representativas estadísticamente se realizó el análisis factorial basado en el análisis de componentes principales ${ }^{7}$.

5 Las obras consultadas para identificar los insumos y productos en la primera etapa del proceso de selección de variables fueron Prieto y Zoflo (2001), Balaguer et al. (2007) y Selva y Sanz (2015).

${ }^{6}$ Los datos de los insumos provienen del Censo Nacional de Gobiernos Municipales y Delegacionales de 2015, que reporta información de 2014. Todos los insumos corresponden a competencias municipales exclusivas. Los datos de los insumos provienen de Finanzas Públicas Estatales y Municipales de 2014 y del Censo Nacional de Gobiernos Municipales y Delegacionales de 2015, reportados por el Inegi.

${ }^{7}$ E1 análisis factorial es una técnica de reducción de datos que determina grupos homogéneos de variables latentes entre numerosas variables observadas. Esos grupos se forman con variables estrechamente correlacionadas, 
El análisis factorial se hizo en tres fases. En la primera se construyó una matriz de correlaciones de Pearson, que elimina las variables de baja correlación con el producto y alta con otros insumos (Zamora, Monroy y Chávez, 2010). En la segunda se aplicaron las pruebas de KaiserMeyer-Olkin (KMO) y de Esfericidad de Bartlett para determinar la confiabilidad del modelo factorial. Por último se calcularon los porcentajes de varianza explicada y la matriz de componentes rotados (Zamora et al., 2010). De este modo, mediante el análisis factorial se determinaron los insumos estadísticamente más representativos de cada producto (ver cuadros 2 a 4 del anexo). Puesto que los resultados arrojaron en forma recurrente dos insumos para los tres productos solo se construyó un modelo DEA (ver cuadro 1).

Cuadro 1

Productos e insumos del modelo DEA

\begin{tabular}{lll}
\hline Productos & Indicador & Unidades \\
\hline Rlumbrado público & Luminarias & $\begin{array}{l}\text { Número de lumina- } \\
\text { rias }\end{array}$ \\
\hline $\begin{array}{l}\text { Seguridad pública mu- } \\
\text { nicipal }\end{array}$ & $\begin{array}{l}\text { Intervenciones de la policía mu- } \\
\text { nicipal }\end{array}$ & $\begin{array}{l}\text { Número de habitan- } \\
\text { tes atendidos de de interven- } \\
\text { ciones }\end{array}$ \\
\hline Insumos & Indicador & Unidades \\
\hline Sueldos y salarios & $\begin{array}{l}\text { Erogación orientada a las re- } \\
\text { muneraciones de empleados y } \\
\text { funcionarios públicos. }\end{array}$ & Pesos mexicanos \\
\hline Gasto de inversión & $\begin{array}{l}\text { Egresos destinados a obra pública } \\
\text { y a incrementar los bienes de } \\
\text { capital. }\end{array}$ & Pesos mexicanos \\
\hline
\end{tabular}

Fuente: Inegi (2015 y 2017), elaboración propia utilizando el programa SPSS.

\section{CARACTERÍSTICAS DEL MODELO DE EFICIENCIA}

Después de revisar la literatura se eligió un modelo DEA con orientación insumo-producto, el cual identifica los cambios equiproporcionales que deben introducir las DMU en los insumos (sueldos y salarios, y gasto de inversión) y en los productos (los servicios) para lograr la eficiencia técnica (Silva et al., 2003). Además, para modelar la eficiencia se adoptó el supuesto de rendimientos variables a escala (VRS), es decir, tomando como referencia una frontera flexible de posibilidades de producción (Herrera y Fracke, 2007), porque los mercados de servicios públicos operan en ambientes sensibles a fallas

procurando que sean independientes (Espejel, González y Perón, 2004; Zamora et al., 2010). 
de mercado, y es posible que los gobiernos municipales no operen en una escala óptima. Así, la comparación entre municipios (las DMU) se realizó entre los que tienen características similares (Balaguer, 2004).

\section{ECUACIÓN DEL MODELO DEA CON VRS}

Después de decidir la orientación y los rendimientos se llegó al siguiente modelo (Silva et al., 2003; Coll y Blasco, 2006):

$\theta^{*}=\operatorname{MAX}(1-K) X,(1+\theta) Y$, sujeto a:

$-y_{i}+Y \lambda \geq 0$

$\theta x_{i}-X \lambda \geq 0$

$n 1^{\prime} \lambda=1$

$\lambda \geq 0$

donde $\theta^{*}$ es la distancia equiproporcional de los insumos y productos a la hipérbola de la envolvente de datos, cuyo valor mide la eficiencia de la unidad de toma de decisión, $y_{i}$ es el vector de productos (servicios públicos), $x_{i}$ es el vector de insumos utilizados, $Y$ es la matriz (mxn) de productos de los 113 municipios, $X$ es la matriz (kxn) de insumos de los 113 municipios y $n 1^{\prime} \lambda=1$ es la restricción que indica una frontera de producción convexa (VRS).

E1 modelo tiene dos restricciones. La primera, $Y \lambda \geq y_{i}$, obliga a la DMU virtual a producir al menos tantos productos como la DMU estudiada; $y$ la segunda, $\theta x i \geq X \lambda$, obliga a que la DMU virtual consuma menor o igual cantidad de insumos que la DMU evaluada. A su vez, la restricción de convexidad, $n 1^{\prime} \lambda=1$, indica la existencia de rendimientos variables a escala (Balaguer, 2004). Por último, el vector de constantes, $\lambda$, permite estimar los pesos que se requieren para que un municipio ineficiente se ubique como eficiente en la frontera de producción (Herrera y Fracke, 2007).

\section{RESULTADOS}

Esta sección presenta los resultados, utilizando el software Efficiency Measurement System (EMS) y entendiendo la eficiencia técnica como la situación en que la reducción de un insumo implica el aumento de otro insumo o la reducción de un producto (Pinzón, 2003).

\section{MUNICIPIOS EFICIENTES}

E1 cuadro 5 del anexo muestra que, en 2014, 22 municipios (el 19,5\%) ejecutaron sus recursos (sueldos y salarios, y gasto de inversión) de manera eficiente para prestar los servicios de alumbrado público, seguridad pública y recolección de basuras. 
Los municipios eficientes se sitúan en el norte y el bajío (mapa 1) y en su mayoría son de carácter semiurbano; con un centro de población principal catalogado como ciudad mediana (La Piedad, Ario, Jiquilpan, Tarímbaro y Zinapécuaro) o en crecimiento (Yurécuaro, Salvador Escalante, Huandacareo, Pajacuarán y Chilchota) (Inegi, 2015).

Una característica común es que todos, salvo Tarímbaro, tienen un número de centros poblacionales inferior al promedio. Además, Tingambato, Huandacareo, Cherán, Chucándiro, Zináparo, Tlazazalca, Chilchota, Yurécuaro, Angangueo, Pajacuarán y Jiménez son eficientes y están entre los 30 municipios con menos centros de población (Inegi, 2010).

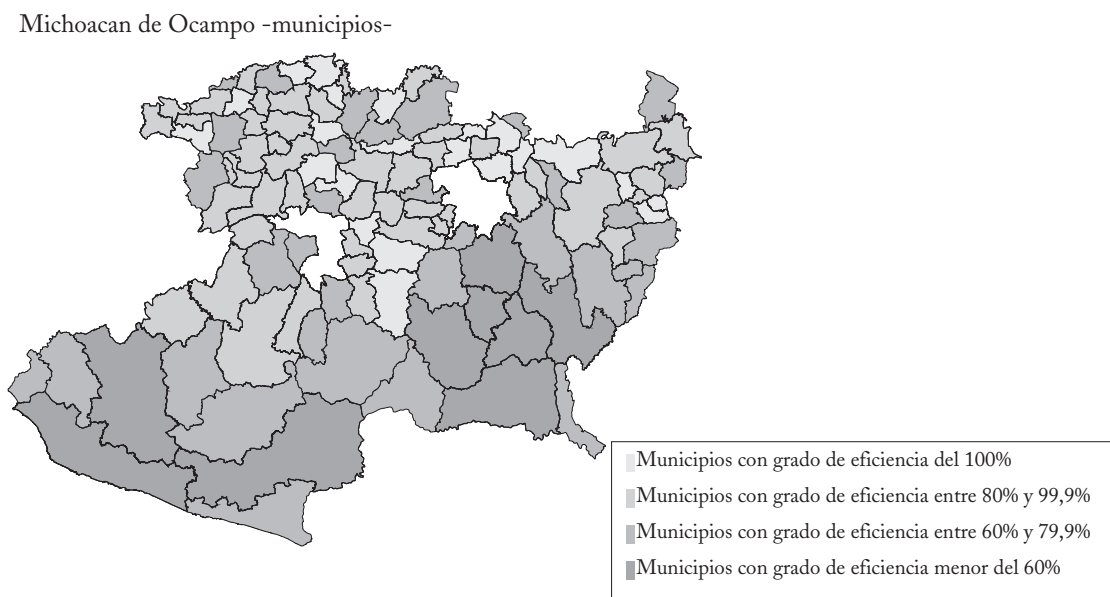

Fuente: Inegi (2015 y 2017), elaboración propia utilizando el programa SPSS.

La mayoría de los municipios eficientes tienen una relación gasto de inversión/gasto corriente más equilibrada que los no eficientes. Otra característica financiera que los distingue es que en el periodo 1990-2014 en casi todos se registró un aumento de ingresos menor al promedio estatal (Inegi, 2017), salvo en Ocampo, Tarímbaro, Cherán y Jiménez (ver cuadros 1 y 5 del anexo).

\section{MUNICIPIOS NO EFICIENTES}

El cuadro 5 del anexo muestra también que 91 municipios no fueron eficientes en 2014. E1 mapa 1 indica que 43 municipios registraron un grado de eficiencia del $80 \%$ al 99,9\%. Es decir, deben modificar equiproporcionalmente sus productos e insumos entre un $20 \%$ y un 0,01\% para ser eficientes. En su mayoría son urbanos o semi-urbanos, 
y con una relación desequilibrada entre gasto corriente y gasto de inversión (Silva et al, 2003).

Por otra parte, 34 municipios tuvieron un grado de eficiencia del $60 \%$ al 79,9\%. Los ingresos de estos municipios aumentaron por encima del promedio entre 1990 y 2014, y su población está diseminada en numerosas comunidades rurales. Entre ellos se destacan Lázaro Cárdenas, Tacámbaro y Chinicuila (Inegi, 2017).

Por último, 9 municipios mostraron un grado de eficiencia inferior al $60 \%$, en su mayoría con una relación gasto de inversión/gasto corriente desequilibrada. Todos ellos son de gran extensión y tienen muchos centros poblacionales (Inegi, 2010).

\section{DisCUSIÓN DE LOS RESULTADOS}

En el periodo 1990-2014, el aumento promedio del ingreso de los municipios eficientes fue de un 637\%, mientras que el de municipios no eficientes fue de 740\% (Inegi, 2017). Estas cifras indican que una mayor disposición de recursos financieros no implica necesariamente un uso más eficiente.

Según datos del Inegi (2017), en los municipios que resultaron eficientes en este estudio el gasto de inversión en 2014 fue proporcionalmente mayor que en los ineficientes. Esto sugiere que el equilibrio entre gasto de inversión y gasto corriente puede favorecer la eficiencia en la provisión de servicios públicos.

Además, la mayoría de los municipios eficientes tenían menos centros de población que el promedio. Los menos eficientes fueron aquellos donde la población habita en pequeñas rancherías (Inegi, 2010). De modo que hay una correlación inversa entre el grado de eficiencia y la dispersión de la población.

Los resultados mencionados coinciden con los argumentos expuestos en diversos trabajos ${ }^{8}$. Así como con el hecho de que una mayor eficiencia en el uso de los recursos permite que los gobiernos locales presten sus servicios de mejor manera y en mayor proporción.

\section{CONCLUSIONES}

La provisión de servicios públicos municipales es un tema que preocupan a la ciudadanía porque incide de manera inmediata en su calidad de vida. Es entonces esencial no concebir la ejecución de los recursos públicos únicamente como una decisión técnica sino como

${ }^{8}$ P. ej., los de Prieto y Zoflo (2001), Balaguer et al. (2007) y Selva y Sanz (2015).

Revista de Economía Institucional, vol. 22, N. ${ }^{\circ} 43$, segundo semestre/2020, Pp. $277^{-299}$ ISSN OI $24-5996 / \mathrm{E}-\mathrm{ISSN} 2346-245^{\circ}$ 
una arena de batalla donde confluyen diversos intereses y puntos de vista que inciden en las decisiones y el ejercicio del gobierno local.

A partir de los trabajos y las concepciones sobre medición de la eficiencia, en este artículo se desarrolló un modelo DEA, con rendimientos variables y una orientación insumo-producto, para determinar qué tan eficientes fueron los municipios de Michoacán en la provisión de servicios públicos en 2014.

Los resultados indican que solo 22 municipios (el 19,5\%) ejecutaron sus recursos financieros de manera eficiente en la prestación de servicios públicos en 2014. Es decir, a pesar del aumento de recursos para los gobiernos municipales su uso y su ejecución son en general ineficientes.

Los resultados sugieren algunas líneas de investigación: 1. Usar el método de fronteras estocásticas (SFA) para calcular la eficiencia; 2. Hacer análisis dinámicos de la eficiencia; 3. Estudiar la eficiencia con diferentes especificaciones de los insumos; 4) Identificar alternativas de política pública para mejorar la eficiencia en los municipios con alta dispersión de la población; 5 . Analizar a profundidad el impacto de la destinación del gasto en la eficiencia; y 6 . Analizar la eficiencia contrastando las cabeceras municipales con las Tenencias y Encargaturas del Orden.

\section{ANEXOS}

Cuadro 1

Aspectos de la provisión de servicios públicos municipales en Michoacán

\begin{tabular}{|c|c|c|c|c|c|c|c|}
\hline \multirow[t]{2}{*}{ Municipio } & \multirow{2}{*}{$\begin{array}{c}\text { Población } \\
2015\end{array}$} & \multicolumn{3}{|c|}{ Evolución de los ingresos municipales } & \multicolumn{3}{|c|}{ Objeto del gasto 2014} \\
\hline & & $\begin{array}{l}\text { Loca- } \\
\text { lidades }\end{array}$ & 1990 & 2014 & Crec, \% & $\begin{array}{l}\text { Gasto de } \\
\text { inversión }\end{array}$ & $\begin{array}{c}\text { Gasto } \\
\text { corriente }\end{array}$ \\
\hline Acuitzio & 11.425 & 54 & $6.297,4$ & $37.196,0$ & 490,6 & $9.946,10$ & $24.626,7$ \\
\hline Aguililla & 15.241 & 639 & $13.642,6$ & $78.720,7$ & 477,1 & $9.985,10$ & $67.212,6$ \\
\hline Álvaro Obregón & 21.651 & 106 & $9.093,1$ & $61.748,4$ & 579,1 & $13.578,10$ & $54.782,1$ \\
\hline Angamacutiro & 15.193 & 43 & $6.862,1$ & $47.195,9$ & 587,7 & $14.521,50$ & $32.674,4$ \\
\hline Angangueo & 11.120 & 32 & $10.104,9$ & $39.795,4$ & 293,8 & $2.367,70$ & $39.183,4$ \\
\hline Apatzingán & 128.250 & 583 & $49.477,7$ & $321.860,1$ & 550,5 & $92.659,90$ & $229.200,2$ \\
\hline Aporo & 3.218 & 16 & $5.841,7$ & $29.700,0$ & 408,4 & $7.517,20$ & $21.212,9$ \\
\hline Aquila & 24.864 & 985 & $12.752,6$ & $126.630,7$ & 892,9 & $52.759,40$ & $73.871,2$ \\
\hline Ario & 36.549 & 232 & $14.466,6$ & $94.366,7$ & 552,3 & $10.467,10$ & $85.755,4$ \\
\hline Arteaga & 22.138 & 909 & $13.547,2$ & $110.894,9$ & 718,5 & $15.955,10$ & $101.552,7$ \\
\hline Briseñas & 11.328 & 14 & $8.933,7$ & $38.220,6$ & 327,8 & $13.145,10$ & $25.075,5$ \\
\hline Buenavista & 47.498 & 458 & $9.359,6$ & $93.879,1$ & 903,1 & $27.196,20$ & $64.665,1$ \\
\hline Carácuaro & 9.485 & 255 & $8.583,6$ & $83.210,7$ & 869,4 & $44.485,70$ & $43.058,9$ \\
\hline Coahuayana & 14.392 & 186 & $6.699,5$ & $43.094,2$ & 543,2 & $9.922,60$ & $33.171,6$ \\
\hline Coalcomán & 18.444 & 1222 & $17.152,5$ & $118.490,6$ & 590,8 & $35.339,60$ & $87.437,9$ \\
\hline Coeneo & 20.749 & 48 & $10.234,1$ & $75.116,4$ & 633,9 & $7.333,60$ & $69.476,8$ \\
\hline Contepec & 34.193 & 121 & $9.966,1$ & $100.283,5$ & 906,2 & $23.419,30$ & $75.553,9$ \\
\hline Copándaro & 9.151 & 16 & $5.308,5$ & $29.801,4$ & 461,3 & $3.980,30$ & $26.464,4$ \\
\hline Cotija & 19.018 & 123 & $12.995,5$ & $76.157,1$ & 486,1 & $24.623,90$ & $55.378,8$ \\
\hline Cuitzeo & 29.681 & 37 & $10.129,3$ & $66.824,9$ & 559,7 & $32.825,50$ & $34.083,3$ \\
\hline Charapan & 12.373 & 18 & $4.747,6$ & $49.455,1$ & 941,6 & $18.575,30$ & $35.374,3$ \\
\hline Charo & 21.784 & 123 & $7.047,1$ & $51.568,2$ & 631,7 & $7.199,20$ & $44.368,9$ \\
\hline Chavinda & 10.258 & 11 & $9.512,1$ & $33.904,6$ & 256,4 & $9.969,10$ & $23.935,4$ \\
\hline
\end{tabular}




\begin{tabular}{|c|c|c|c|c|c|c|c|}
\hline \multirow[t]{2}{*}{ Municipio } & \multirow{2}{*}{$\begin{array}{c}\text { Población } \\
2015\end{array}$} & \multicolumn{3}{|c|}{ Evolución de los ingresos municipales } & \multicolumn{3}{|c|}{ Objeto del gasto 2014} \\
\hline & & $\begin{array}{l}\text { Locali- } \\
\text { dades }\end{array}$ & 1990 & 2014 & Crec, \% & $\begin{array}{l}\text { Gasto de } \\
\text { inversión }\end{array}$ & $\begin{array}{c}\text { Gasto } \\
\text { corriente }\end{array}$ \\
\hline Cherán & 19.081 & 23 & $6.043,2$ & $55.392,9$ & 816,6 & $15.859,30$ & $39.533,5$ \\
\hline Chilchota & 39.035 & 30 & $14.340,1$ & $84.607,6$ & 490,1 & $28.857,10$ & $55.750,4$ \\
\hline Chinicuila & 5.032 & 391 & $1.960,8$ & $58.745,5$ & 2895,9 & $27.847,80$ & $33.549,6$ \\
\hline Chucándiro & 4.559 & 23 & $4.908,5$ & $29.296,1$ & 496,8 & $4.802,30$ & $28.633,2$ \\
\hline Churintzio & 5.016 & 21 & $6.098,6$ & $41.246,1$ & 576,3 & $22.082,10$ & $19.164,1$ \\
\hline Churumuco & 15.455 & 205 & $7.130,9$ & $63.398,2$ & 789,1 & $27.923,40$ & $35.474,8$ \\
\hline Ecuandureo & 12.788 & 35 & $10.720,4$ & $47.473,9$ & 342,8 & $5,871,9$ & $46.301,7$ \\
\hline Epitacio Huerta & 16.622 & 111 & $6.505,5$ & $63.539,3$ & 876,6 & $29.506,90$ & $34.032,6$ \\
\hline Erongarícuaro & 15.291 & 28 & $5.362,1$ & $58.478,0$ & 990,6 & $6.157,20$ & $62.078,7$ \\
\hline Gabriel Zamora & 22.707 & 89 & $7.870,1$ & $62.238,7$ & 690,8 & $24.397,50$ & $37.841,1$ \\
\hline Hidalgo & 122.619 & 552 & $37.035,5$ & $305.837,3$ & 725,7 & $45.477,20$ & $248.304,7$ \\
\hline La Huacana & 34.243 & 325 & $14.684,7$ & $123.316,5$ & 739,7 & $16.015,70$ & $116.092,7$ \\
\hline Huandacareo & 11.723 & 18 & $10.308,8$ & $34.467,7$ & 234,3 & $6.931,30$ & $29.066,1$ \\
\hline Huaniqueo & 8.093 & 36 & $6.076,8$ & $34.146,7$ & 461,9 & $9.413,40$ & $24.733,2$ \\
\hline Huetamo & 40.818 & 601 & $26.641,6$ & $146.562,6$ & 450,1 & $62.312,50$ & $66.451,3$ \\
\hline Huiramba & 8.838 & 21 & $5.953,7$ & $27.460,1$ & 361,2 & $7.100,10$ & $21.728,3$ \\
\hline Indaparapeo & 16.990 & 69 & $6.386,7$ & $45.718,4$ & 615,8 & $8.691,10$ & $39.056,4$ \\
\hline Irimbo & 15.215 & 65 & $7.025,2$ & $49.739,2$ & 6,08 & $13.183,70$ & $35.095,3$ \\
\hline Ixtlán & 12.720 & 44 & $8.495,5$ & $42.627,6$ & 401,7 & $8.983,60$ & $32.187,1$ \\
\hline Jacona & 69.744 & 100 & $22.738,7$ & $174.979,1$ & 669,5 & $41.335,70$ & $133.643,3$ \\
\hline Jiménez & 12.426 & 34 & $8.316,3$ & $94.328,4$ & 1034,2 & $53.064,30$ & $40.736,2$ \\
\hline Jiquilpan & 32.950 & 68 & $25.774,4$ & $108.834,6$ & 322,2 & $36.508,80$ & $74.317,6$ \\
\hline Juárez & 14 & 63 & $7.911,6$ & $44.163,8$ & 458,2 & $15.941,20$ & $29.603,3$ \\
\hline Jungapeo & 21.548 & 142 & $7.191,5$ & $66.011,8$ & 817,9 & $27.496,80$ & $38.107,3$ \\
\hline Lagunillas & 5.571 & 12 & $4.552,4$ & $30.496,6$ & 569,9 & $7.547,10$ & $28.059,9$ \\
\hline Madero & 18.030 & 606 & $13.047,7$ & $85.452,3$ & 554,9 & $21.321,60$ & $66.495,2$ \\
\hline Maravatío & 88.535 & 221 & $10.692,6$ & $231.492,9$ & 2064,9 & $96.992,90$ & $134.217,1$ \\
\hline Marcos Castellanos & 13.750 & 53 & $21.649,8$ & $58.052,7$ & 168,1 & $30.559,10$ & $26.880,5$ \\
\hline Lázaro Cárdenas & 183.185 & 541 & $19.121,5$ & $658.219,5$ & 3342,2 & $99.171,30$ & $517.203,2$ \\
\hline Morelia & 784.776 & 544 & $271.414,1$ & $2.211,3$ & 714,7 & $481.017,70$ & $1.950,9$ \\
\hline Morelos & 7.806 & 33 & $5.776,9$ & $40.719,6$ & 604,8 & $15.658,10$ & $23.704,7$ \\
\hline Múgica & 45.484 & 91 & $12.417,5$ & $119.647,9$ & 863,5 & $48.632,60$ & $71.015,2$ \\
\hline Nahuatzen & 28.074 & 10 & $7.103,5$ & $62.180,6$ & 775,3 & $19.276,10$ & $42.904,6$ \\
\hline Nocupétaro & 8.195 & 361 & $8.751,4$ & $63.023,8$ & 620,1 & $9.782,30$ & $52.604,3$ \\
\hline N. Parangaricutiro & 19.595 & 89 & $6.734,9$ & $56.891,3$ & 744,7 & $18.483,30$ & $41.190,4$ \\
\hline Nuevo Urecho & 7.988 & 67 & $4.778,1$ & $35.939,0$ & 652,1 & $15.350,40$ & $20.588,5$ \\
\hline Numarán & 9.794 & 33 & $4.696,5$ & $33.596,5$ & 615,3 & $7.922,30$ & $31.899,2$ \\
\hline Ocampo & 24.424 & 52 & $9.126,0$ & $68.831,9$ & 654,2 & $49.127,40$ & $67.955,1$ \\
\hline Pajacuarán & 19.440 & 32 & $6.910,4$ & $58.366,1$ & 744,6 & $12.125,50$ & $46.658,6$ \\
\hline Panindícuaro & 15.705 & 42 & $6.921,6$ & $105.558,6$ & 1425,1 & $34.396,50$ & $111.179,2$ \\
\hline Parácuaro & 37.464 & 130 & $7.726,5$ & $79.873,7$ & 933,7 & $31.829,90$ & $51.771,8$ \\
\hline Paracho & 26.789 & 54 & $8.470,9$ & $77.768,9$ & 818,1 & $23.269,60$ & $54.499,3$ \\
\hline Pátzcuaro & 93.265 & 144 & $24.490,9$ & $185.195,2$ & 656,1 & $43.862,30$ & $141.332,8$ \\
\hline Penjamillo & 16.920 & 83 & $8.247,1$ & $65.039,2$ & 688,6 & $26.838,10$ & $38.642,9$ \\
\hline Peribán & 27.832 & 138 & $7.963,7$ & $56.946,6$ & 615,1 & $10.281,20$ & $47.000,6$ \\
\hline La Piedad & 103.702 & 183 & $97.590,8$ & $238.583,0$ & 144,4 & $42.780,40$ & $195.802,6$ \\
\hline Purépero & 15.255 & 30 & $9.997,6$ & $47.821,2$ & 378,3 & $10.808,90$ & $37.012,2$ \\
\hline Puruándiro & 67.644 & 103 & $23.413,5$ & $233.642,5$ & 897,8 & $96.451,60$ & $137.190,9$ \\
\hline Queréndaro & 13.836 & 65 & $6.593,6$ & $69.203,8$ & 949,5 & $4.685,40$ & $73.644,3$ \\
\hline Quiroga & 27.862 & 29 & $9.554,4$ & $78.531,4$ & 721,9 & $29.601,40$ & $49.932,3$ \\
\hline Cojumatlán & 10.45 & 20 & $6.483,3$ & $44.543,1$ & 587,1 & $23.108,70$ & $21.434,2$ \\
\hline Los Reyes & 69.723 & 38 & $24.239,5$ & $146.049,6$ & 502,1 & $39.681,60$ & $112.825,4$ \\
\hline Sahuayo & 76.587 & 56 & $48.089,1$ & $276.023,8$ & 473,9 & $95.137,40$ & $159.415,4$ \\
\hline San Lucas & 17.845 & 129 & $7.427,6$ & $63.008,0$ & 748,2 & $24.823,30$ & $39.615,1$ \\
\hline Santa Ana Maya & 12.466 & 29 & $7.521,7$ & $41.138,0$ & 446,9 & $11.019,10$ & $31.433,1$ \\
\hline Salvador Escalante & 49.588 & 124 & $15.054,2$ & $126.085,5$ & 737,5 & $51.676,50$ & $118.088,3$ \\
\hline Senguio & 19.146 & 89 & $4.974,9$ & $65.465,6$ & 1215,9 & $25.788,70$ & $39.676,8$ \\
\hline Susupuato & 8.804 & 147 & $5.454,4$ & $48.103,2$ & 781,9 & $6.660,70$ & $40.808,8$ \\
\hline Tacámbaro & 76.661 & 338 & $5.913,8$ & $179.132,9$ & 2929,1 & $32.509,70$ & $132.473,7$ \\
\hline Tancítaro & 31.1 & 180 & $8.455,6$ & $75.267,4$ & 790,1 & $26.655,40$ & $48.612,2$ \\
\hline Tangamandapio & 29.268 & 40 & $6.329,4$ & $72.877,3$ & 1051,3 & $17.070,70$ & $54.425,5$ \\
\hline Tangancícuaro & 33.621 & 101 & $14.131,9$ & $81.103,4$ & 473,9 & $19.740,90$ & $56.393,6$ \\
\hline Tanhuato & 15.352 & 27 & $6.887,5$ & $60.363,0$ & 776,4 & $28.029,50$ & $34.716,2$ \\
\hline Taretan & 14.819 & 39 & $6.030,7$ & $33.751,9$ & 459,6 & $3.656,70$ & $31.548,1$ \\
\hline Tarímbaro & 105.4 & 169 & $8.685,1$ & $174.277,4$ & 1906,6 & $78.424,80$ & $92.100,8$ \\
\hline Tepalcatepec & 23.842 & 167 & $17.175,6$ & $58.185,5$ & 238,7 & $9.406,80$ & $48.790,9$ \\
\hline
\end{tabular}




\begin{tabular}{|c|c|c|c|c|c|c|c|}
\hline \multirow[t]{2}{*}{ Municipio } & \multirow{2}{*}{$\begin{array}{c}\text { Población } \\
2015\end{array}$} & \multicolumn{3}{|c|}{ Evolución de los ingresos municipales } & \multicolumn{3}{|c|}{ Objeto del gasto 2014} \\
\hline & & $\begin{array}{c}\text { Locali- } \\
\text { dades }\end{array}$ & 1990 & 2014 & Crec, $\%$ & $\begin{array}{l}\text { Gasto de } \\
\text { inversión }\end{array}$ & $\begin{array}{c}\text { Gasto } \\
\text { corriente }\end{array}$ \\
\hline Tingambato & 15.01 & 17 & $4.768,4$ & $32, .615,1$ & 583,9 & $6.318,60$ & $26.296,4$ \\
\hline Tingüindín & 14.532 & 33 & $4.873,1$ & $48.998,5$ & 905,49 & $16.124,60$ & $37.088,3$ \\
\hline Tiquicheo & 13.731 & 669 & $9.715,1$ & $85.673,3$ & 781,8 & $23.588,60$ & $62.081,6$ \\
\hline Tlalpujahua & 27.788 & 81 & $12.356,2$ & $66.982,9$ & 442,1 & $17.415,50$ & $49.567,3$ \\
\hline Tlazazalca & 6.202 & 27 & $5.818,5$ & $50.443,4$ & 766,9 & $20.587,40$ & $40.726,3$ \\
\hline Tocumbo & 11.994 & 120 & $6.910,4$ & $41.764,2$ & 504,3 & $13.034,90$ & $30.752,9$ \\
\hline Tumbiscatío & 6.947 & 614 & $9.726,2$ & $50.573,6$ & 419,9 & $11.717,20$ & $43.795,1$ \\
\hline Turicato & 31.849 & 767 & $14.350,2$ & $134.904,6$ & 840,1 & $31.004,60$ & $104.888,1$ \\
\hline Tuxpan & 27.371 & 120 & $39.388,6$ & $139.863,1$ & 255,1 & $80.649,50$ & $54.986,3$ \\
\hline Tuzantla & 15.383 & 433 & $9.375,7$ & $77.360,8$ & 725,1 & $16.481,40$ & $68.772,7$ \\
\hline Tzintzuntzan & 14.432 & 42 & $5.473,9$ & $43.965,5$ & 703,1 & $15.027,00$ & $28.758,9$ \\
\hline Tzitzio & 8.966 & 737 & $9.135,7$ & $63.985,7$ & 600,3 & $3.823,30$ & $61.929,8$ \\
\hline Uruapan & 334.749 & 462 & $104.773,9$ & $752.117,8$ & 617,8 & $179.891,10$ & $565.814,5$ \\
\hline Venustiano Carranza & 24.708 & 33 & $13.548,5$ & $61.614,7$ & 354,7 & $15.625,20$ & $48.737,6$ \\
\hline Villamar & 17.166 & 57 & $7.892,3$ & $51.190,6$ & 548,6 & $13.388,80$ & $39.482,6$ \\
\hline Vista Hermosa & 20.624 & 23 & $7.951,1$ & $43.494,6$ & 447,1 & $7.217,70$ & $36.276,8$ \\
\hline Yurécuaro & 31.404 & 30 & $11.363,4$ & $89.059,5$ & 683,7 & $36.942,60$ & $52.116,8$ \\
\hline Zacapu & 75.632 & 100 & $32.801,1$ & $173.767,7$ & 429,7 & $63.981,20$ & $104.820,1$ \\
\hline Zamora & 196.208 & 200 & $92.930,5$ & $426.139,0$ & 358,5 & $82.145,10$ & $343.273,4$ \\
\hline Zináparo & 3.2 & 24 & $5.533,1$ & $21.125,2$ & 281,8 & $7.305,50$ & $13.819,6$ \\
\hline Zinapécuaro & 47.327 & 154 & $15.834,5$ & $129.376,8$ & 717,1 & $34, .950,1$ & $105.386,7$ \\
\hline Ziracuaretiro & 17.394 & 41 & $5.321,1$ & $42.962,9$ & 707,4 & $8.380,40$ & $32.549,2$ \\
\hline Zitácuaro & 164 & 630 & $42.682,2$ & $423.055,0$ & 891,1 & $44.699,40$ & $368.234,6$ \\
\hline José Sixto Verduzco & 26.214 & 44 & $7.176,9$ & $82.554,5$ & 1050,2 & $26.923,90$ & $56.342,3$ \\
\hline
\end{tabular}

Fuente: Inegi (2015 y 2017) e Inafed (2015), elaboración propia.

Cuadro 2

Luminarias

\begin{tabular}{|c|c|c|c|c|c|c|c|}
\hline \multicolumn{8}{|c|}{ Matriz de correlaciones } \\
\hline & & EM & $\mathrm{SP}$ & GP & BI & GI & $\mathrm{RF}$ \\
\hline \multirow[t]{6}{*}{ Correlación } & EM & 1 & 0,957 & 0,838 & 0,272 & 0,089 & 0,135 \\
\hline & $\mathrm{SP}$ & 9,57 & 1 & 0,914 & 0,241 & 0,061 & 0,18 \\
\hline & GP & 0,838 & 0,914 & 1 & 0,126 & 0,12 & 0,178 \\
\hline & BI & 0,272 & 0,241 & 0,126 & 1 & $-0,025$ & $-0,028$ \\
\hline & GI & 0,089 & 0,061 & 0,12 & $-0,025$ & 1 & 0,285 \\
\hline & RF & 0,135 & 0,18 & 0,178 & $-0,028$ & 0,285 & 1 \\
\hline \multicolumn{8}{|c|}{ KMO y prueba de Bartlett } \\
\hline \multirow{2}{*}{\multicolumn{7}{|c|}{$\begin{array}{l}\text { Medida de adecuación muestral de Kaiser-Meyer-Olkin } \\
\text { Chi-cuadrado aproximado }\end{array}$}} & 0,613 \\
\hline & & & & & & & 517,288 \\
\hline \multirow{2}{*}{\multicolumn{6}{|c|}{ Prueba de esfericidad de Bartlett }} & G1 & 15 \\
\hline & & & & & & Sig. & 00 \\
\hline
\end{tabular}

\begin{tabular}{lcc}
\hline Matriz de componentes rotados & \multicolumn{2}{c}{ Componente } \\
\hline & 1 & 2 \\
\hline EM & 0,957 & $-0,112$ \\
SP & 0,981 & $-0,094$ \\
GP & 0,931 & $-0,011$ \\
BI & 0,311 & $-0,355$ \\
GI & 0,167 & 0,761 \\
RF & 0,263 & 0,733 \\
\hline
\end{tabular}

Nota: $\mathrm{EM}=$ Número de empleados, $\mathrm{SP}=$ Servicios personales, $\mathrm{GP}=$ Gasto per cápita, $\mathrm{BI}=$ Número de bienes inmuebles, $\mathrm{GI}=$ Gasto de inversión, $y \mathrm{RF}=$ Recursos financieros.

Fuente: Inegi (2015 y 2017), elaboración propia utilizando el programa SPSS. 
Cuadro 3

Recolección de basuras

Matriz de correlaciones

\begin{tabular}{|c|c|c|c|c|c|c|}
\hline \multirow{6}{*}{ Correlación } & & GI & SP & $\mathrm{BI}$ & $\mathrm{RF}$ & EM \\
\hline & GI & 1 & 0,061 & $-0,025$ & 0,285 & 0,089 \\
\hline & SP & 0,061 & 1 & 0,241 & 0,180 & 0,957 \\
\hline & BI & $-0,025$ & 0,241 & 1 & $-0,028$ & 0,272 \\
\hline & RF & 0,285 & 0,180 & $-0,028$ & 1 & 0,135 \\
\hline & EM & 0,089 & 0,957 & 0,272 & 0,135 & 1 \\
\hline \multicolumn{7}{|c|}{ KMO y prueba de Bartlett } \\
\hline \multirow{4}{*}{\multicolumn{5}{|c|}{$\begin{array}{l}\text { Medida de adecuación muestral de Kaiser-Meyer-Olkin } \\
\text { Chi-cuadrado aproximado } \\
\text { Prueba de esfericidad de Bartlett }\end{array}$}} & & 0.511 \\
\hline & & & & & & 298.845 \\
\hline & & & & & G1 & 100 \\
\hline & & & & & Sig. & 00 \\
\hline \multicolumn{7}{|c|}{ Matriz de componentes rotados } \\
\hline & & \multicolumn{5}{|c|}{ Componente } \\
\hline \multirow{2}{*}{\multicolumn{3}{|c|}{$\overline{\mathrm{GI}}$}} & 1 & & \multicolumn{2}{|c|}{2} \\
\hline & & & 0,193 & & \multicolumn{2}{|c|}{$0, \overline{758}$} \\
\hline \multicolumn{2}{|l|}{ SP } & & 0,955 & & \multicolumn{2}{|c|}{$-0,102$} \\
\hline \multicolumn{2}{|l|}{ BI } & & 0,420 & & \multicolumn{2}{|c|}{$-0,366$} \\
\hline \multicolumn{2}{|l|}{ RF } & & 0,303 & & \multirow{2}{*}{\multicolumn{2}{|c|}{$\begin{array}{c}0,728 \\
-0,121\end{array}$}} \\
\hline \multicolumn{2}{|l|}{ EM } & & 0,957 & & & \\
\hline
\end{tabular}

Nota: GI = Gasto de inversión, $\mathrm{SP}=$ Servicios personales, $\mathrm{BI}=$ Número de bienes inmueble, $\mathrm{RF}=$ Recursos financieros, y EM = Número de empleados.

Fuente: Inegi (2015 y 2017), elaboración propia utilizando el programa SPSS.

Cuadro 4

Intervenciones de la policía

Matriz de correlaciones

\begin{tabular}{lcccccc}
\hline & & GI & EM & SP & GP & BI \\
\hline Correlación & GI & 1 & 0,089 & 0,061 & 0,120 & $-0,025$ \\
& EM & 0,089 & 1 & 0,957 & 0,838 & 0,272 \\
& SP & 0,061 & 0,957 & 1 & 0,914 & 0,241 \\
& GP & 0,120 & 0,838 & 0,914 & 1 & 0,126 \\
& BI & $-0,025$ & 0,272 & 0,241 & 0,126 & 1 \\
\hline
\end{tabular}

KMO y prueba de Bartlett

Medida de adecuación muestral de Kaiser-Meyer-Olkin.

Chi-cuadrado aproximado

Prueba de esfericidad de Bartlett

0.633

Prueba de esfericidad de Bartlett

Matriz de componentes rotados

\begin{tabular}{ccc}
\hline & \multicolumn{2}{c}{ Componente } \\
\cline { 2 - 3 } & 1 & 2 \\
\hline GI & 0,132 & 0,844 \\
SP & 0,964 & $-0,017$ \\
GP & 0,985 & $-0,015$ \\
BI & 0,933 & 0,107 \\
\hline
\end{tabular}

Nota: $\mathrm{GI}=$ Gasto de inversión, $\mathrm{EM}=$ Número de empleados, $\mathrm{SP}=$ Servicios personales, $\mathrm{GP}=$ Gasto per cápita, y $\mathrm{BI}=$ Número de bienes inmuebles.

Fuente: Inegi (2015 y 2017), elaboración propia utilizando el programa SPSS. 
Cuadro 5

Modelo DEA para estimar la eficiencia en la provisión de servicios municipales

\begin{tabular}{|c|c|c|c|c|c|c|}
\hline DMU & & Productos & & Insu & mos & Nivel \\
\hline Municipio & $\begin{array}{l}\text { Alumbra- } \\
\text { do público }\end{array}$ & $\begin{array}{l}\text { Interven- } \\
\text { ciones de } \\
\text { la policía } \\
\text { municipal }\end{array}$ & $\begin{array}{c}\text { Limpieza } \\
\text { y recolec- } \\
\text { ción de } \\
\text { basuras }\end{array}$ & $\begin{array}{l}\text { Gasto de } \\
\text { inversión }\end{array}$ & $\begin{array}{c}\text { Servicios } \\
\text { personales }\end{array}$ & $\begin{array}{c}\text { Eficiencia } \\
(\%)\end{array}$ \\
\hline Acuitzio & 1.236 & 88 & 7.998 & $9.946,1$ & $14.270,5$ & 76,7 \\
\hline Aguililla & 1.672 & 85 & 15.241 & $9.985,1$ & $35.754,5$ & 66,2 \\
\hline Álvaro Obregón & 1.715 & 1.026 & 21.651 & $3.578,1$ & $16.962,9$ & 100 \\
\hline Angamacutiro & 2.970 & 93 & 3.039 & $14.521,5$ & $12.468,6$ & 100 \\
\hline Angangueo & 794 & 1.200 & 11.120 & $2.367,7$ & $16.406,3$ & 100 \\
\hline Apatzingán & 9.793 & 6.089 & 128.250 & $92.659,9$ & $127.229,9$ & 98,1 \\
\hline Aporo & 291 & 47 & 3.057 & $7.517,2$ & $12.044,8$ & 80,1 \\
\hline Aquila & 3.300 & 300 & 14.918 & $52.759,4$ & $42.696,5$ & 55,7 \\
\hline Ario & 3.800 & 900 & 36.549 & $10.467,1$ & $39.861,1$ & 100 \\
\hline Arteaga & 2.114 & 240 & 22.138 & $15.955,1$ & $48.834,3$ & 56,9 \\
\hline Briseñas & 1.000 & 230 & 9.062 & $13.145,1$ & $13.651,2$ & 78,8 \\
\hline Buenavista & 5.026 & 151 & 37.998 & $27.196,2$ & $32.739,2$ & 98,2 \\
\hline Carácuaro & 1.191 & 73 & 9.485 & $44.485,7$ & $17.141,7$ & 56,5 \\
\hline Coahuayana & 1.680 & 163 & 11.514 & $9.922,6$ & $18.929,2$ & 67,7 \\
\hline Coalcomán & 1.500 & 145 & 16.600 & $35.339,6$ & $40.918,1$ & 42.3 \\
\hline Coeneo & 2.555 & 212 & 20.749 & $7.333,6$ & $25.155,1$ & 94,7 \\
\hline Contepec & 1.866 & 222 & 23.935 & $23.419,3$ & $18.433,6$ & 89,6 \\
\hline Copándaro & 971 & 100 & 9.151 & $3.980,3$ & $13.854,3$ & 97,1 \\
\hline Cotija & 2.652 & 165 & 19.018 & $24.623,9$ & $23.867,9$ & 74,5 \\
\hline Cuitzeo & 3.906 & 82 & 29.681 & $32.825,5$ & $17.943,5$ & 100 \\
\hline Charapan & 1.300 & 58 & 12.373 & $18.575,3$ & $12.669,6$ & 83,7 \\
\hline Charo & 2.300 & 745 & 21.784 & $7.199,2$ & $18.013,5$ & 99,2 \\
\hline Chavinda & 1.573 & 53 & 5.129 & $9.969,1$ & $12.252,7$ & 80,9 \\
\hline Cherán & 848 & 44 & 17.936 & $15.859,3$ & $11.416,5$ & 100 \\
\hline Chilchota & 2.331 & 1.020 & 39.035 & $28.857,1$ & $23.824,1$ & 100 \\
\hline Chinicuila & 615 & 52 & 5.032 & $27.847,8$ & $11.989,3$ & 64,9 \\
\hline Chucándiro & 1.800 & 137 & 912 & $4.802,4$ & $9.723,9$ & 100 \\
\hline Churintzio & 1.320 & 45 & 4.013 & $22.082,1$ & $8.180,6$ & 89,2 \\
\hline Churumuco & 1.321 & 88 & 14.682 & $27.923,4$ & $16.597,3$ & 73,4 \\
\hline Ecuandureo & 1.922 & 226 & 12.788 & $5.871,9$ & $16.257,3$ & 89,5 \\
\hline Epitacio Huerta & 936 & 700 & 16.622 & $29,506,9$ & $19.978,2$ & 77,5 \\
\hline Erongarícuaro & 1.035 & 218 & 10.704 & $6.157,2$ & $15.601,3$ & 82,5 \\
\hline Gabriel Zamora & 2.291 & 142 & 22.707 & $24.397,5$ & $23.597,8$ & 76,7 \\
\hline Hidalgo & 7.440 & 1.715 & 122.619 & $45.477,2$ & $141.744,4$ & 84,6 \\
\hline La Huacana & 1.150 & 152 & 32.531 & $16.015,7$ & $43.754,8$ & 63,7 \\
\hline Huandacareo & 1.413 & 44 & 11.723 & $6.931,3$ & $10.909,1$ & 100 \\
\hline Huaniqueo & 1.444 & 73 & 8.093 & $9.413,4$ & $10.816,8$ & 92,4 \\
\hline Huetamo & 850 & 172 & 6.123 & $62.312,5$ & $59.573,7$ & 51,9 \\
\hline Huiramba & 958 & 170 & 7.512 & $7.100,7$ & $11.349,2$ & 93,1 \\
\hline Indaparapeo & 1.106 & 190 & 16.990 & $8.691,4$ & $18.245,7$ & 82,4 \\
\hline Irimbo & 3.000 & 758 & 15.215 & $13.183,7$ & $15.469,9$ & 100 \\
\hline Ixtlán & 1.961 & 523 & 12.084 & $8.983,6$ & $14.778,2$ & 90,6 \\
\hline Jacona & 4.000 & 2.223 & 69.744 & $41.335,7$ & $82.924,6$ & 88,5 \\
\hline Jiménez & 1.941 & 1.383 & 4.349 & $53.064,3$ & $18.223,1$ & 100 \\
\hline Jiquilpan & 7.800 & 334 & 31.303 & $36.508,8$ & $40.218,9$ & 100 \\
\hline Juárez & 850 & 134 & 7.194 & $15.941,2$ & $13.200,9$ & 73,5 \\
\hline Jungapeo & 1.700 & 44 & 21.548 & $27.496,8$ & $18.857,1$ & 82,5 \\
\hline Lagunillas & 800 & 82 & 5.571 & $7.547,1$ & $10.592,8$ & 92,9 \\
\hline Madero & 1.117 & 423 & 3.606 & $21.321,6$ & $26.178,1$ & 47,3 \\
\hline Maravatío & 5.704 & 942 & 88.535 & $96.992,9$ & $61.200,7$ & 93,8 \\
\hline Marcos Castellanos & 1.390 & 64 & 13.750 & $30.559,5$ & $10.633,5$ & 93,2 \\
\hline Lázaro Cárdenas & 15.414 & 2.478 & 128.230 & $99.171,3$ & $333.792,1$ & 70,5 \\
\hline
\end{tabular}

Revista de Economía Institucional, vol. 22, N. ${ }^{\circ} 43$, segundo semestre/2020, Pp. $277-299$ ISSN OI $24-5996 / \mathrm{E}-$ ISSN $2346-2450$ 


\begin{tabular}{|c|c|c|c|c|c|c|}
\hline DMU & & Productos & & Insu & imos & Nivel \\
\hline Municipio & $\begin{array}{l}\text { Alumbra- } \\
\text { do público }\end{array}$ & $\begin{array}{l}\text { Interven- } \\
\text { ciones de } \\
\text { la policía } \\
\text { municipal }\end{array}$ & $\begin{array}{c}\text { Limpieza } \\
\text { y recolec- } \\
\text { ción de } \\
\text { basuras }\end{array}$ & $\begin{array}{l}\text { Gasto de } \\
\text { inversión }\end{array}$ & $\begin{array}{c}\text { Servicios } \\
\text { personales }\end{array}$ & $\begin{array}{c}\text { Eficiencia } \\
\text { (\%) }\end{array}$ \\
\hline Morelia & 65.361 & 3.109 & 706.298 & $481.017,7$ & $555.772,4$ & 1,3 \\
\hline Morelos & 2.106 & 43 & 7.806 & $15.658,1$ & $13.097,6$ & 82,8 \\
\hline Múgica & 1.700 & 683 & 31.839 & $48.632,6$ & $46.979,5$ & 70,1 \\
\hline Nahuatzen & 1.400 & 428 & 25.267 & $19.276,5$ & $23.287,9$ & 82,2 \\
\hline Nocupétaro & 1.300 & 85 & 1.393 & $9.782,3$ & $19.968,2$ & 52,3 \\
\hline N. Parangaricutiro & 968 & 150 & 18.027 & $18.483,3$ & $19.386,5$ & 75,2 \\
\hline Nuevo Urecho & 655 & 72 & 7.988 & $15.350,4$ & $11.800,7$ & 80,1 \\
\hline Numarán & 1.903 & 158 & 2.155 & $7.922,3$ & $12.480,8$ & 86,9 \\
\hline Ocampo & 2.300 & 210 & 19.539 & $49.127,4$ & $10.983,6$ & 100 \\
\hline Pajacuarán & 2.300 & 211 & 19.440 & $12.125,5$ & $14.028,9$ & 100 \\
\hline Panindícuaro & 2.300 & 318 & 15.705 & $34.396,5$ & $19.800,5$ & 73,4 \\
\hline Parácuaro & 3.200 & 60 & 22.478 & $31.829,9$ & $23.337,9$ & 80,9 \\
\hline Paracho & 2.130 & 260 & 21.431 & $23.269,6$ & $23.768,5$ & 74,7 \\
\hline Pátzcuaro & 7.397 & 2576 & 83.939 & $43.862,3$ & $89.642,5$ & 97,9 \\
\hline Penjamillo & 1.502 & 321 & 14.382 & $26.838,1$ & $17.029,8$ & 74,9 \\
\hline Peribán & 2.700 & 75 & 27.832 & $10.281,2$ & $25.899,3$ & 91,9 \\
\hline La Piedad & 9.949 & 2.082 & 103.702 & $42.780,4$ & $97.482,4$ & 100 \\
\hline Purépero & 2.362 & 79 & 15.255 & $10.808,9$ & $21.839,3$ & 78,8 \\
\hline Puruándiro & 6.838 & 1.408 & 20.293 & $96.451,6$ & $76.299,8$ & 79,4 \\
\hline Queréndaro & 1.320 & 100 & 13.836 & $4.685,4$ & $19.938,3$ & 78,3 \\
\hline Quiroga & 1.972 & 145 & 27.862 & $29.601,4$ & $25.820,9$ & 79,3 \\
\hline Cojumatlán & 1.441 & 146 & 10.241 & $23.108,7$ & $10.139,5$ & 89,7 \\
\hline Los Reyes & 7.000 & 105 & 62.751 & $39.681,6$ & $53.837,8$ & 97,8 \\
\hline Sahuayo & 7.194 & 358 & 76.587 & $95.137,4$ & $69.216,5$ & 85,2 \\
\hline San Lucas & 1.692 & 118 & 14.276 & $24.823,3$ & $21.661,2$ & 63,1 \\
\hline Santa Ana Maya & 1.566 & 40 & 12.466 & $11.019,1$ & $15.658,1$ & 78,3 \\
\hline Salvador Escalante & 2.820 & 86 & 49.588 & $11.676,5$ & $30.422,8$ & 100 \\
\hline Senguio & 1.298 & 120 & 19.146 & $25.788,7$ & $15.476,6$ & 86,7 \\
\hline Susupuato & 832 & 72 & 8.804 & $6.660,7$ & $16.248,3$ & 76,2 \\
\hline Tacámbaro & 3.645 & 162 & 19.932 & $32.509,7$ & $75.882,8$ & 76,7 \\
\hline Tancítaro & 2.793 & 194 & 27.990 & $26.655,4$ & $41.539,2$ & 66,2 \\
\hline Tangamandapio & 1.780 & 211 & 29.268 & $17.070,7$ & $25.090,9$ & 84,7 \\
\hline Tangancícuaro & 3.518 & 129 & 16.811 & $19.740,9$ & $30.991,8$ & 80,2 \\
\hline Tanhuato & 1.477 & 82 & 10.746 & $28.029,5$ & $15.733,9$ & 67,7 \\
\hline Taretan & 1.302 & 187 & 14.078 & $3.656,7$ & $14.986,4$ & 99,8 \\
\hline Tarímbaro & 8.038 & 837 & 105.400 & $78.424,8$ & $62.899,2$ & 100 \\
\hline Tepalcatepec & 2.550 & 788 & 23.842 & $9.406,8$ & $28.160,4$ & 88,7 \\
\hline Tingambato & 2.400 & 194 & 13.509 & $6.318,6$ & $12.053,5$ & 100 \\
\hline Tingüindín & 2.124 & 202 & 11.626 & $16.124,6$ & $14.956,5$ & 80,3 \\
\hline Tiquicheo & 1.113 & 82 & 11.534 & $23.588,6$ & $39.066,5$ & 36,3 \\
\hline Tlalpujahua & 2.000 & 208 & 27.788 & $17.415,5$ & $29.512,4$ & 75,3 \\
\hline Tlazazalca & 2.060 & 170 & 6.202 & $20.587,4$ & $7.213,5$ & 100 \\
\hline Tocumbo & 1.355 & 91 & 11.994 & $13.034,9$ & $13.173,8$ & 84,3 \\
\hline Tumbiscatío & 1.300 & 144 & 6.947 & $11.717,2$ & $15.576,4$ & 68,1 \\
\hline Turicato & 2.230 & 622 & 19.109 & $31.004,6$ & $39.319,2$ & 56,8 \\
\hline Tuxpan & 2.500 & 180 & 21.897 & $80.649,5$ & $22.692,6$ & 72,6 \\
\hline Tuzantla & 1.500 & 130 & 15.383 & $16.481,4$ & $19.497,4$ & 71,6 \\
\hline Tzintzuntzan & 1.600 & 118 & 14.432 & $15.027,1$ & $13.290,6$ & 88,9 \\
\hline Tzitzio & 2.220 & 140 & $15.666,80$ & $3.823,3$ & $18.431,5$ & 73,1 \\
\hline Uruapan & 19.001 & 4.559 & 334.749 & $179.891,3$ & $293.842,3$ & 2,9 \\
\hline Venustiano Carranza & 2.348 & 146 & 24.708 & $15.625,2$ & $18.030,7$ & 84,9 \\
\hline Villamar & 1.700 & 380 & 13.733 & $13.388,8$ & $18.248,9$ & 74,8 \\
\hline Vista Hermosa & 1.927 & 502 & 20.624 & $7.217,7$ & $17.923,6$ & 92,5 \\
\hline Yurécuaro & 2.413 & 1.369 & 31.404 & $36.942,6$ & $24.511,3$ & 100 \\
\hline Zacapu & 5.000 & 1.250 & 75.632 & $63.981,2$ & $73.199,7$ & 84,3 \\
\hline Zamora & 14.182 & 3.714 & 196.208 & $82.145,1$ & $194.534,5$ & 91,5 \\
\hline
\end{tabular}




\begin{tabular}{lcccccc}
\hline DMU & \multicolumn{5}{c}{ Productos } & \multicolumn{2}{c}{ Insumos } & Nivel \\
\hline Municipio & $\begin{array}{c}\text { Alumbra- } \\
\text { do público }\end{array}$ & $\begin{array}{c}\text { Interven- } \\
\text { ciones de } \\
\text { la policía } \\
\text { municipal }\end{array}$ & $\begin{array}{c}\text { Limpieza } \\
\text { y recolec- } \\
\text { ción de } \\
\text { basuras }\end{array}$ & $\begin{array}{c}\text { Gasto de } \\
\text { inversión }\end{array}$ & $\begin{array}{c}\text { Servicios } \\
\text { personales }\end{array}$ & $\begin{array}{c}\text { Eficiencia } \\
(\%)\end{array}$ \\
\hline Zináparo & 976 & 40 & 1.600 & $7.305,5$ & $8.510,9$ & 100 \\
Zinapécuaro & 6.056 & 1.321 & 42.594 & $34.950,9$ & $43.291,9$ & 100 \\
Ziracuaretiro & 1.452 & 186 & 17.394 & $8.380,4$ & $13.766,6$ & 98,4 \\
Zitácuaro & 8.008 & 3.187 & 49.243 & $44.699,4$ & $175.853,9$ & 75,5 \\
José Sixto Verduzco & 2.220 & 938 & 11.796 & $26.923,9$ & $22.552,4$ & 83,5 \\
\hline
\end{tabular}

Fuente: Inegi (2015 y 2017), elaboración propia utilizando el programa SPSS.

\section{REFERENCIAS BIBLIOGRÁFICAS}

Aberbach, J. y Christensen, T. (2005). Ciudadanos y consumidores: E1 dilema. Public Management Review, 7(2), 225-245.

Afonso, A. y Fernandes, S. (2006). Measuring local government spending efficiency: Evidence for the Lisbon region. Regional Studies, 4O(2), 39-53.

Aigner, D., Lovell, C. et al. (1977). Formulation and estimation of stochastic frontier production function models. Journal of Econometrics, 6(1), 21-37.

Andrade, E. (2006). Derecho Municipal. México DF: Oxford University Press.

Antúnez, I. y Galilea, S. (2003). Servicios públicos urbanos y gestión local en América Latina: problemas, metodologias y politicas. Chile: Cepal.

Astudillo, M. (2005). La coordinación fiscal en México: logros y desafíos. Problemas del Desarrollo, 36(141), 205-212.

Autoridad Superior de Michoacán (ASM). (2011). Manual por Objeto del Gasto, [http://www.secfinanzas.michoacan.gob.mx/download/ presupuesto_de_egresos_del_estado_2017/Anexo-Descriptivo-Clasificador-por-Objeto-del-Gasto.pdf]

Balaguer-C., M. (2004). La eficiencia en las administraciones locales ante diferentes medidas de output. Hacienda Pública Española, 170(3), 37-58.

Balaguer-C., M., Prior, D. et al. (2007). On the determinants of local government performance: A two-stage nonparametric approach. European Economic Review, 51(2), 425-451.

Bellido, A. (2010). Análisis de la Eficiencia Municipal: La municipalidad de la Plata. Argentina: Universidad Nacional de la Plata.

Bemowski, K. (1991). The benchmarking bandwagon. Quality Progress, 24(1), 19-24.

Bosch, E., Navarro, A. et al. (1999). Eficiencia técnica y asignativa en la distribución de la energía eléctrica. El caso de EPE SF. Asociación Argentina de Economía Pública, 1-24.

Bradford, D., Malt, R. et al. (1969). The rising cost of local public services: Some evidence and reflections. National Tax Journal, 22(2), 185-202.

Broid, D. (2010). La evolución del predial en México: Los incentivos cruzados de la descentralización fiscal y política, 1990-2007. Finanzas públicas, 2(3-4), 69-192.

Budnick, F. (2007). Matemáticas aplicadas para administración, economía y ciencias sociales. México: Mc Graw Hill. 
Cabrero, E. y Arellano, D. (2011). Los gobiernos municipales a debate. Un análisis de la institución municipal a través de la encuesta Inegi 2009. México DF: CIDE.

Charnes, A., Cooper, W. et al. (1978). Measuring the efficiency of decision making units. European Journal of Operational Research, 2(6), 429-444.

Coll, V. y Blasco, O. (2006). Evaluación de la eficiencia mediante el análisis envolvente de datos: introducción a los modelos básicos. Madrid: Universidad de Valencia.

De Borger, B., Kerstens, K. et al. (1994). Explaining differences in productive efficiency: An application to Belgian municipalities. Public Choice, 80(3-4), 339-358.

De Borger, B. y Kerstens, K. (1996). Cost efficiency of Belgian local governments: A comparative analysis of FDH, DEA, and econometric approaches. Regional Science and Urban Economics, 26(2), 145-170.

Delfín, O. y Navarro, J. (2014). La Eficiencia de los Puertos en México. México DF: Universidad Michoacana de San Nicolás de Hidalgo.

Denhardt, R. y Denhardt, J. (2003). The New Public Service: Serving, not Steering. Nueva York: M. E. Sharp.

Espejel, A., González, I. et al. (2004). El índice de deterioro ambiental en los municipios de Tlaxcala: Una propuesta metodológica. Gaceta ecológica, 70, 19-30.

Farrell, M. (1957). The measurement of productive efficiency. Journal of the Royal Statistical Society Series A (General), 120(3), 253-281.

Fernández, J. (2002). Servicios Público Municipales. México DF: UNAM.

García, R. y Gómez, P. (2011). Servicios Públicos en los gobiernos locales: Un análisis a través de las encuestas municipales. En E. Cabrero (ed.), Los gobiernos municipales a debate. Un análisis de la institución municipal a través de la encuesta Inegi 2009 (pp. 157-213). México DF: CIDE.

Giménez, V. y Prior, D. (2003). Evaluación frontera de la eficiencia en costes. Aplicación a los municipios de Cataluña. Papeles de Economía Española, 95, 113-124.

Gómez, R. (2014). Medición de la eficiencia de las bancas comercial y de desarrollo con la metodología DEA (Data Envelopment Analysis), a partir de los procesos de internacionalización del sistema bancario en México. México DF: Instituto Politécnico Nacional.

H. Congreso de la Unión. (1978). Ley Nacional de Coordinación Fiscal. Congreso de la Unión, [http://www.diputados.gob.mx/LeyesBiblio/ ref/1cf/LCF_orig_27dic78_ima.pdf]

H. Congreso de la Unión. (2019). Constitución Política de los Estados Unidos Mexicanos. Congreso de la Unión, [http://www.diputados. gob.mx/LeyesBiblio/htm/1.htm]

H. Congreso de Michoacán. (2001). Ley Orgánica Municipal del Estado de Michoacán de Ocampo, [https://docs.mexico.justia.com/estatales/ michoacan/ley-organica-municipal-del-estado-de-michoacan-deocampo.pdf].

H. Congreso de Michoacán. (2019). Constitución Política del Estado Libre y Soberano de Michoacán de Ocampo, [http://www.senado. gob.mx/AHyML/index_htm_files/ConstitMichoacan.pdf]. 
Herrera, P. y Fracke, P. (2007). Análisis de la eficiencia del gasto municipal y sus determinantes. Perú: Pontifica Universidad Católica del Perú.

Herrera, P. y Málaga, R. (2007). Indicadores de desempeño y análisis de eficiencia de los municipios peruanos: Una aproximación no paramétrica en un contexto de descentralización. Perú: CIES.

Herrera, V., Maya, S. et al. (2017). Diagnóstico de las Haciendas Públicas Locales en México. México DF: Senado de la República.

Instituto del Desarrollo Municipal (IDM). (1999). E1 ABC de los Gobiernos Municipales, [https://bibliotecavirtualceug.files.wordpress. com/2017/06/a-b-c-del-gobierno-municipal.pdf].

Instituto Nacional de Estadística Geografía e Informática (Inegi). (2010). Censo General de Población y Vivienda 2000, [https://www.inegi. org. $\mathrm{mx} /$ programas/ccpv/2000/].

Inegi (2015). Censo Nacional de Gobiernos Municipales y Delegacionales. [https://www.inegi.org.mx/programas/cngmd/2015/]

Inegi (2017). Estadísticas de Finanzas Públicas Estatales y Municipales, [https://www.inegi.org. mx/programas/finanzas/default. html\#Tabulados]

Instituto Nacional para el Federalismo y el Desarrollo Municipal (Inafed). (2015). Egresos de los gobiernos Municipales, Federalismo Hacendario. [http://www.inafed.gob.mx/es/inafed/Municipales].

Jaramillo, R., Monroy, G. et al. (2005). La encrucijada de los Servicios Públicos. Bogotá: Grupo Editorial Norma.

Moore, A., Nolan, J. et al. (2005). Putting out the trash. Measuring municipal services efficiency in U.S. Cities. Urban Affairs Review, 41(2), 237-259.

Navarro, J. (2005). La eficiencia del sector eléctrico en México. México DF: Universidad Michoacana de San Nicolás de Hidalgo

Navarro, J., Ayvar, F. et al. (2016). Generación de bienestar social en México: Un estudio DEA a partir del IDH. Economía, Sociedad y Territorio, 16(52), 591-621.

Oates, W. (1999). An essay on fiscal federalism. Journal of Economics Literature, 37(3), 1120-1149.

Olivera, D. y Cano, M. (2012). La evaluación del desempeño a nivel municipal. Revista Ciencia Administrativa, 2, 117-121.

Olson, M. (1969). The principle of fiscal equivalence: The division of responsibilities among different levels of government. American Economic Review, 59(2), 479-487.

Osuna, J. y Márquez, C. (2013). Guía para la evaluación de políticas públicas. Instituto de Desarrollo Regional, [http://coeval.morelos. gob.mx/Acervo-Bibliografico/Libro-GuiaEvaluacionPoliticasPublicas].

Pacheco, F., Sánchez, R. y Villlena, M. (2013). Eficiencia de los gobiernos locales y sus determinantes, [http://www.dipres.gob.cl/598/ articles-114713_doc_pdf.pdf].

Pinzón, M. (2003). Medición de eficiencia técnica relativa en hospitales públicos de baja complejidad mediante la metodología data envelopment analysis (DEA). [https://ideas.repec.org/p/col/000118/003192. html]. 
Prieto, A. y Zoflo, J. (2001). Evaluating effectiveness in public provision of infrastructure and equipment: The case of Spanish municipalities. Journal of Productivity Analysis, 15(1), 41-58.

Raich, U. (2000). Desempeño financiero municipal. Algunas propuestas de evaluación. Hacienda Municipal, 20(7), 84-90.

Restrepo, M. y Villegas, J. (2008). Análisis envolvente de datos: introducción $y$ herramienta pública para su utilización, [https://juangvillegas.files. wordpress.com/2013/08/restrepo-villegas-dea.pdf].

Seiford, L. y Thrall, R. (1990). Recent developments in DEA: The mathematical programming approach to frontier analysis. Journal of Econometrics, 46(1-2), 7-38.

Seijas, A. (2005). Análisis de la eficiencia técnica en la educación secundaria. Estudios de Economía Aplicada, 23(2), 299-322.

Selva, C. y Sanz, M. (2015). La eficiencia municipal en la prestación de bienes y servicios públicos: De la medición de su nivel al análisis de sus posibles causas. International journal of maritime history, 27(4), 157-170.

Silva, M., Castro, P. y Thanassoulis, E. (2003). Finding closest targets in non-oriented DEA models: The case of convex and non-convex technologies. Journal of Productivity Analysis, 19(2-3), 251-269.

Starling, R. (1988). Strategies for Policy making. Estados Unidos: The Dorsey Press.

Taïrou, A. (2000). Does inefficiency explain financial vulnerability of French municipalities?. En International Conference on Accounting, Auditing y Management in Public Sector Reforms. Zaragoza: EIASM.

Valencia, S. (2006). Derecho municipal. México DF: Porrúa.

Vanden, P., Tulkens, H. y Jamar, M. (1993). Cost efficiency in Belgian municipalities. En H. Fried, C. Knox y S. Schmidt (eds.), The measurement of productive efficiency - Techniques and applications (pp. 300-334). Nueva York: Oxford University Press.

Worthington, A. y Dollery, B. (2000). Efficiency measurement in the local public sector: Econometric and mathematical programming frontier techniques, [https://core.ac.uk/download/pdf/6438618.pdf].

Zamora, S., Monroy, L. y Chávez, C. (2010). Análisis factorial: una técnica para evaluar la dimensionalidad de las pruebas, [http://www.ceneval.edu. $\mathrm{mx} /$ documents/20182/0/CuadernoTecnico061aed.pdf/a922a295-93f3$4 b d 0-9 c 8 b-28 a 52 c 499 c f 5]$. 